\title{
Hemofilia A adquirida associada à artrite reumatoide
}

\author{
Marlene Freire ${ }^{1}$, Reginaldo Botelho Teodoro ${ }^{2}$, Dulcinéia Aparecida Nogueira ${ }^{3}$, \\ Danielle Patrícia Castanheira Rita ${ }^{4}$, Elmiro Ribeiro Filho ${ }^{5}$ Ĺ́lian Vieira de Sousa ${ }^{6}$
}

\begin{abstract}
RESUMO
O aparecimento do anticorpo contra fator VIII é um fenômeno bem estabelecido na hemofilia A, ocorrendo em 5 a $15 \%$ dos pacientes hemofílicos dos Estados Unidos, Inglaterra, Suécia e França. Nos pacientes não-hemofílicos o seu aparecimento é raro, podendo ocorrer em indivíduos saudáveis, principalmente idosos e mulheres no puerpério, pacientes com neoplasia maligna ou doenças autoimunes, como lúpus eritematoso sistêmico, artrite reumatoide e síndrome de Sjögren. Descrevemos o caso de uma paciente de 64 anos de idade, portadora de artrite reumatoide soropositiva há 23 anos, que há 10 dias desenvolveu equimoses e hematomas progressivos cuja investigação foi compatível com a presença de anticorpo contra fator VIII. Foi instituída terapia com metilprednisolona, ciclofosfamida endovenosa, imunoglobulina e reposição de complexo protrombínico, com remissão do quadro hemorrágico e negativação do anticorpo contra o fator VIII. Concluímos com esse caso que, apesar de ser rara, a presença de inibidores adquiridos do fator VIII deve ser pesquisada quando pacientes portadores de doença autoimune desenvolvem manifestações hemorrágicas associadas ao prolongamento do TTPA com TAP e contagem plaquetária normais.
\end{abstract}

Palavras-chave: artrite reumatoide, inibidor do fator VIII, hemofilia A adquirida.

\section{INTRODUÇÃO}

A hemofilia A adquirida é uma alteração rara da coagulação sanguínea caracterizada pelo aparecimento de anticorpos circulantes contra a atividade pró-coagulante do fator VIII. ${ }^{1}$ Essa condição pode ocorrer em associação com doenças autoimunes, como artrite reumatoide (AR), ${ }^{1-8}$ lúpus eritematoso sistêmico $(\mathrm{LES})^{2,3}$ e síndrome de Sjögren. ${ }^{9}$ A hemofilia A adquirida também tem sido relatada em idosos sadios, ${ }^{7,8}$ mulheres no pós-parto ${ }^{8}$ e pacientes com neoplasia maligna, ${ }^{2}$ além de ser relacionada ao uso de fármacos como a penicilina. ${ }^{3,8}$

Várias terapias incluindo corticosteroide, ciclofosfamida, ciclosporina ou vincristina têm sido usadas para reduzir ou eliminar os inibidores do fator VIII. Tratamentos com altas doses de imunoglobulina, interferon e plasmaférese têm sido relatados, porém atualmente não se tem definida nenhuma terapia "padrão ouro", ${ }^{4,510} \mathrm{O}$ esquema de tratamento mais utilizado quando da associação com doenças autoimunes consiste na combinação da metilprednisolona e ciclofosfamida., ${ }^{4,5,710,11}$ Nos últimos anos, tem sido citado que o rituximabe poderá ser um valioso agente no tratamento da hemofilia adquirida. ${ }^{11}$

Descrevemos o caso de uma paciente com AR que apresentou quadro hemorrágico grave com diagnóstico de hemofilia A adquirida.

Recebido em 10/04/2008. Aprovado, após revisão, em 11/01/2009. Declaramos a inexistência de conflitos de interesse.

Trabalho realizado na disciplina de Reumatologia e Hematologia do Departamento de Clínica Médica da Universidade Federal do Triângulo Mineiro (UFTM) - Uberaba, MG.

1. Professor Adjunto da Disciplina de Reumatologia - UFTM

2. Professor Assistente da Disciplina de Reumatologia - UFTM

3. Médico Assistente da Disciplina de Hematologia - UFTM

4. Residente (R2) em Reumatologia - UFTM

5. Residente (R2) em Hematologia - UFTM

6. Residente (R2) em Hematologia - UFTM

Endereço para correspondência: Marlene Freire. Departamento de Clínica Médica, Disciplina de Reumatologia. Rua Getúlio Guaritá, sem número, bairro Abadia. CEP 38025-110. Uberaba, MG. E-mail: m.freire@terra.com.br 


\section{RELATO DE CASO}

A paciente tem 64 anos de idade, é parda, viúva, natural de Ibiá ( $\mathrm{mg}$ ) e procedente de Frutal ( mg). Tem história de equimoses e hematomas progressivos por todo corpo há 10 dias. Foi admitida pela primeira vez no Hospital Escola da Universidade Federal do Triângulo Mineiro (UFTM) em abril de 2005. Apresentava diagnóstico de AR há 23 anos e fazia uso de prednisona $5 \mathrm{mg} /$ dia desde o início da doença, em acompanhamento facultativo. No exame físico, encontramos equimoses e hematomas extensos em membros inferiores (Figuras 1 e 2), abdome e vulva, além das mãos com desvio ulnar dos dedos e espessamento sinovial nas articulações metacarpofalangianas.

Os exames laboratoriais mostraram hemácias de $2.370 .000 /$ $\mathrm{mm}^{3}$, hemoglobina de $7,4 \mathrm{~g} / \mathrm{dL}$, hematócrito de $22,4 \%$, plaquetas de $340.000 / \mathrm{mm}^{3}$, proteína C reativa de $23,3 \mathrm{mg} / \mathrm{dL}$, $\alpha 1$-glicoproteína ácida de $1,0 \mathrm{~g} / \mathrm{dL}$, velocidade de hemossedimentação de $50 \mathrm{~mm}$ na primeira hora, tempo de ativação de protrombina (TAP) com $78 \%$ de atividade e tempo de tromboplastina parcial ativada (TTPA) com relação paciente-teste de 3,45 (normal de 0,9 a 1,25). A dosagem de fator reumatoide foi de 51,6 UI $/ \mathrm{mL}$ (valor de referência $<10 \mathrm{UI} / \mathrm{mL}$ ). A dosagem de fator VIII foi de $4,3 \%$ (valor de referência 50 a $150 \%$ ) e a dosagem do inibidor de fator VIII foi maior que 10 UI Bethesda. Diante do alargamento do TTPA, da redução da dosagem do fator VIII e da presença de anticorpo contra o fator VIII, foi feito o diagnóstico de hemofilia A adquirida (moderada intensidade $=$ nível de Fator VIII entre 1 e 5\%).

$\mathrm{O}$ tratamento durante a internação consistiu na realização via endovenosa de três ciclos de metilprednisolona $700 \mathrm{mg} /$ dia, dois ciclos de $25 \mathrm{~g}$ de imunoglobulina, ciclofosfamida $500 \mathrm{mg}$ com intervalo de 21 dias entre eles e reposição de complexo protrombínico (Fator VIII 1250 UI de 12/12 horas por 15 dias).

A paciente evoluiu com melhora importante do quadro clínico, normalização do TTPA e negativação do anticorpo contra o fator VIII em um mês de tratamento. Recebeu alta em uso de prednisona $100 \mathrm{mg} /$ dia e foi submetida a mais três ciclos mensais de ciclofosfamida $500 \mathrm{mg}$ via endovenosa no Ambulatório de Reumatologia. Em agosto de 2005, foi suspensa a ciclofosfamida, permanecendo a paciente em acompanhamento no ambulatório com evolução favorável.

\section{DISCUSSÃO}

O relato do caso mostra uma paciente com doença autoimune que desenvolveu hemofilia adquirida e foi tratada com sucesso com uma combinação de metilprednisolona, imunoglobulina, reposição de fator VIII/complexo protrombínico e ciclofosfamida endovenosa.

O diagnóstico diferencial de equimoses e/ou hematomas cursando com alargamento do TTPA, a redução da dosagem do fator VIII e a presença do inibidor de fator VIII caracterizando a hemofilia adquirida inclui neoplasias ou doenças autoimunes como AR, LES e síndrome de Sjögren, uso de drogas como penicilina, mulheres no pós-parto e idosos sadios.

A associação de AR com o anticorpo antifator VIII circulante é rara. Os inibidores do fator VIII em pacientes não-hemofílicos são geralmente associados a sangramento severo, que pode levar à morte em mais de $20 \%$ dos casos. ${ }^{1,4} \mathrm{O}$ principal objetivo do tratamento da hemofilia adquirida, além de controlar as hemorragias, é erradicar o anticorpo contra o fator VIII. ${ }^{10}$

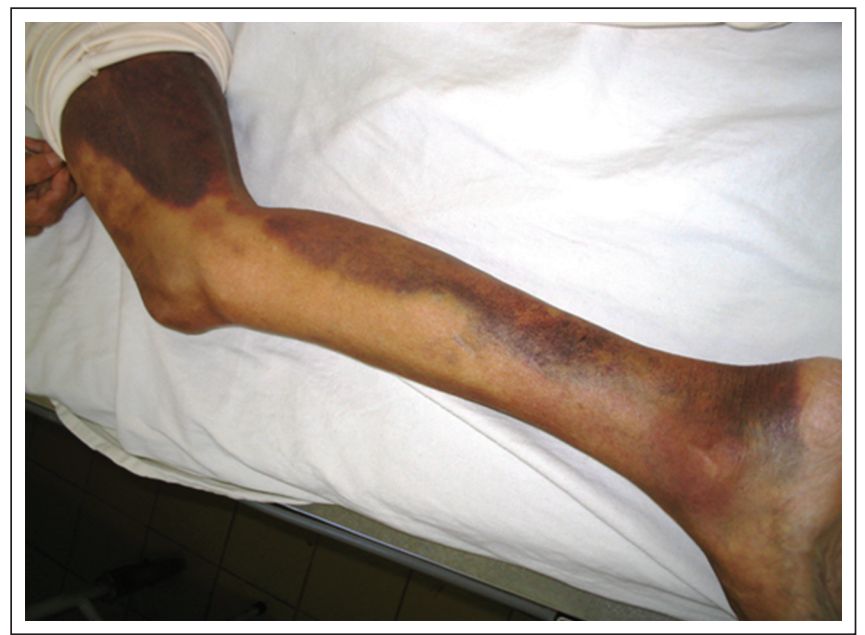

Figura 1. Hematoma e equimose extensa de membro inferior.

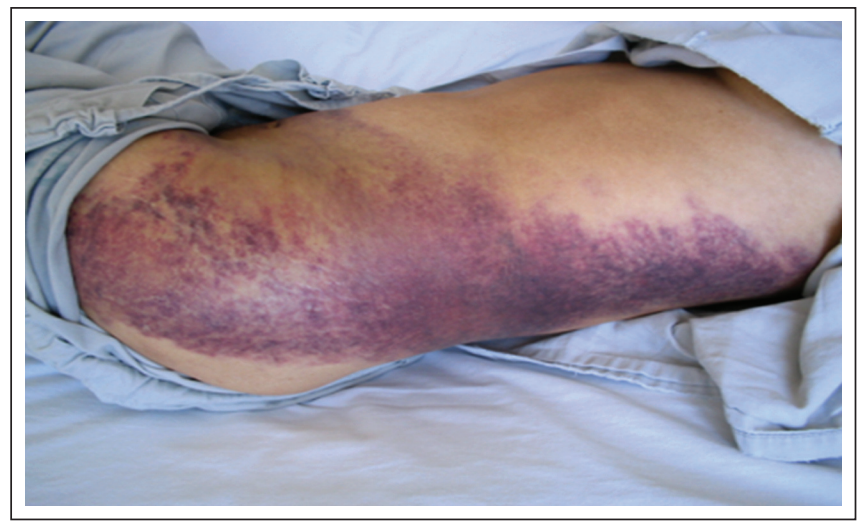

Figura 2. Equimose de membro inferior. 
O tipo, a extensão e a gravidade da hemorragia em pacientes com autoanticorpos (hemofilia adquirida) diferem da observada em pacientes com hemofilia A e que desenvolvem inibidores. Nesses pacientes, os sangramentos se iniciam na infância e ocorrem nas articulações, nos músculos e em tecidos moles, sendo que a presença de inibidores do fator VIII não aumenta a frequência de episódios hemorrágicos, mas dificulta o controle de tais eventos. Já os pacientes com hemofilia adquirida são habitualmente adultos com hemorragias súbitas, mais graves na pele, em tecido subcutâneo e nos músculos; as hemartroses são menos comuns. ${ }^{10,11}$
O fato de a produção de anticorpo antifator VIII não estar associada à atividade da doença, como observado no caso descrito, sugere a importância de monitorar a coagulação sanguínea nos pacientes em remissão clínica e com manifestações hemorrágicas. O diagnóstico precoce é de suma importância, pois, apesar de rara, essa condição é muito grave e com alta mortalidade., ${ }^{3,46}$

Concluímos com a apresentação desse caso que a pesquisa de inibidores adquiridos contra o fator VIII deve ser realizada em pacientes com doença autoimune que desenvolvem manifestações hemorrágicas na vigência de TTPA prolongado com TAP e contagem plaquetária normais. 


\section{REFERÊNCIAS}

\section{REFERENCES}

1. Soriano R mg, Matthews JM, Guerado-Parra E. Case Report. Acquired haemophilia and rheumatoid arthritis. Br J Rheumatol 1987;26:381-383.
2. Hall RL, Lealy MF. Acquired Factor VIII autoantibody: four cases demonstrating the heterogenous nature of this condition and problems involved in diagnosis and treatment Factor VIII Inhibitors in Rheumatoid Arthritis. Eur J Haematol 2001;66:206-209.

3. Nishino Y, Ueki K, Suto M, Uchiumi H, Ota F, Tamura S et al. Successful treatment of patients with rheumatic disorders and acquired factor VIII inhibitors with cyclophosphamide and prednisolone combination therapy: two case reports. J Int Med Res 2001;29:432-463.

4. Pignone A, Matucci-Cerinic M, Morfini M, Lombardi A, Ferrini Rossi PL, Cagnoni M. Suppression of autoantibodies to factor VIII and correction of factor VIII deficiency with a combined steroidcyclophosphamide-porcine factor VIII treatment in a patient with rheumatoid arthritis. J Intern Med 1992;231:617-619.

5. Collins D, Bourke BE. Haemophilia and Rheumatoid Arthritis. Br J Rheumatol 1988;4:332.

6. Struillou L, Fiks-Sigaud M, Barrier J, Blat E. Acquired haemophilia and rheumatoid arthritits: success of immunoglobulin therapy. J Intern Med 1993;233(3):304-5.

7. Vignes S, Le Moing V, MeeKel P, Papo T, Wechsler B, Godeau P. Acquired hemophilia: A rare complication of Sjogren's syndrome. Clin Exp Rheumatol 1996;14:559-560.

8. Kueh YK, Koh DR, Chan TB, Han P. Acquired factor VIIIC deficiency due to circulating factor VIIIC inhibitors. Ann Acad Med Singapore 1988;17(4):589-94.

9. Raphael N, Shulman, Hirschman RJ. Acquired Hemophilia. Trans Assoc Am Physicians 1969;82:388-97.

10. Cabral A, Romão T, Monteiro JA, Uva LS. Hemofilia Adquirida, a propósito de um caso clínico. Med Inter 1998;5(1):53-59.

11. Giangrande P. Hemofilia Adquirida. Tratamiento de la Hemofilia 2005;38:1-8. 